\title{
Bayer Corneal Epitheliopathy Grade 3
}

National Cancer Institute

\section{Source}

National Cancer Institute. Bayer Corneal Epitheliopathy Grade 3. NCI Thesaurus. Code C128443.

Corneal ulcer without risk of acute rupture; central stromal opacity. 